\title{
Interdisziplinäre Anforderungsmuster für smarte persönliche Assistenten
}

\section{Mittel zu Erfassung divergenter Anforderungen aus Informatik und Recht}

Bei der Entwicklung smarter persönlicher Assistenten können Anforderungsmuster helfen, die oftmals konfliktären Anforderungen zu systematisieren und miteinander

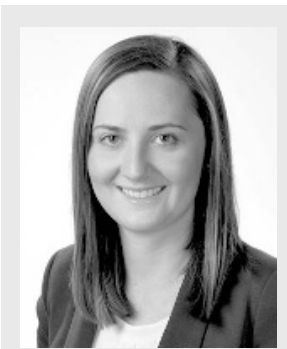

\section{Ernestine Dickhaut, M.Sc.}

Doktorandin und Wissenschaftliche Mitarbeiterin am Fachgebiet für Wirtschaftsinformatik und dem Wissenschaftlichen Zentrum für Informationstechnikgestaltung (ITeG) an der Universität Kassel.

E-Mail: ernestine.dickhaut@uni-kassel.de

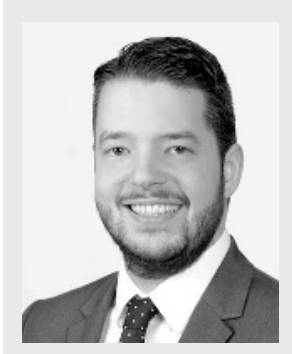

\section{Dr. Andreas Janson}

Forschungsgruppenleiter und Postdoktorand am Fachgebiet für Wirtschaftsinformatik und dem Wissenschaftlichen Zentrum für Informationstechnikgestaltung (ITeG) an der Universität Kassel. E-Mail: andreas.janson@uni-kassel.de

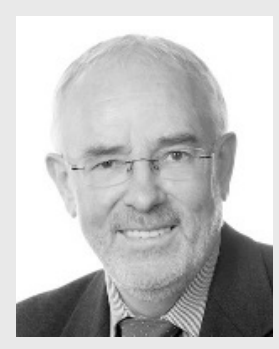

\section{Prof. Dr. Alexander Roßnagel}

Wiss. Leiter der Projektgruppe verfassungsverträgliche Technikgestaltung (provet) und Direktor des Forschungszentrums für Informationstechnik-Gestaltung (ITeg) an der Universität Kassel. E-Mail: a.rossnagel@uni-kassel.de

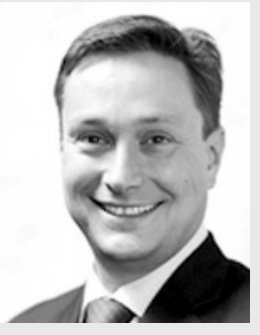

\section{Prof. Dr. Jan Marco Leimeister}

Leiter des Fachgebietes Wirtschaftsinformatik und Direktor am Wissenschaftlichen Zentrum für Informationstechnikgestaltung (ITeG) an der Universität Kassel. Ordinarius für Wirtschaftsinformatik und Direktor am Institut für Wirtschaftsinformatik (IWI-HSG), Universität St. Gallen, Schweiz. E-Mail: leimeister@uni-kassel.de in Einklang zu bringen. Sie können wiederkehrende rechtliche und qualitative Anforderungen in einer für Entwickler verständlichen Form darstellen und so helfen, sie im Entwicklungsprozess besser zu berücksichtigen.

\section{Zielkonflikte}

Für Systeme der Informationstechnik, die wie smarte persönliche Assistenten (SPA) im Alltag breit genutzt werden, sind in der Entwicklung viele verschiedene Anforderungen aus unterschiedlichen Bereichen zu beachten, beispielsweise aus rechtlicher oder betriebswirtschaftlicher Sicht. So ist die Dienstleistungsqualität, also die Qualität der Leistungserbringung, ein entscheidender betriebswirtschaftlicher Faktor für die Zufriedenheit mit dem System. So könnte beispielsweise ein Nutzer ${ }^{1}$ eines SPAs, der auf Basis der eigenen Präferenzen in der Lage ist, personalisierte Dienste auszuführen, einem SPA, der eine solche Personalisierung nicht zulässt, vorziehen. Denn Personalisierung der Funktionalitäten ist dabei ein Faktor für Dienstleistungsqualität und somit für Nutzer ein Indikator, wie zufrieden sie mit dem System während der Nutzung voraussichtlich sein werden. ${ }^{2}$ Das Konzept der Rechtsverträglichkeit dagegen verfolgt das Ziel, den Nutzer der Technik bestmöglich und langfristig vor den Risiken der Techniknutzung zu schützen. ${ }^{3}$ Um dieses Ziel zu erreichen, dürfen, bei einer rechtsverträglichen Systemgestaltung, nur so viele personenbezogene Daten der Nutzer verarbeitet werden, wie erforderlich.

\footnotetext{
* Beitrag zum DFG-Forschungsprojekt "Anforderungs- und Entwurfsmuster zur rechtsverträglichen und qualitätszentrierten Entwicklung kontextsensitiver Applikationen" (AnEkA), https://www.uni-kassel.de/fb07/institute/iwr/personenfachgebiete/rossnagel-prof-dr/forschung/provet/aneka.html

1 Zur besseren Lesbarkeit des Textes wird auf die Aufzählung mehrerer Geschlechter verzichtet. Der Begriff "Nutzer" und ähnliche Begriffe umfassen immer auch alle Personen anderen Geschlechts.

2 Lin/Hsieh, Assessing the Self-service Technology Encounters: Development and Validation of SSTQUAL Scale, in Journal of Retailing 2011, 194-206.

3 Siehe näher Thies/Knote/Jandt/Söllner, in diesem Heft.
} 
Demnach stehen die Anforderungen der Dienstleistungsqualität oftmals denen der Rechtsverträglichkeit diametral entgegen. Diese zu erfassen, sie zu bewerten und zu gewichten und sie bei vielfältigen Zielkonflikten - in optimaler Weise in der Systementwicklung zu berücksichtigen, ist äußerst schwierig. Wenn es aber nicht gelingt, sie in den Systementwicklungsprozess zu integrieren, werden sie ignoriert und führen zu Problemen mit der Akzeptanz oder Akzeptabilität des entwickelten Systems. Sie nachträglich zu berücksichtigen erweist sich vielfach als äußerst schwierig und teuer. Jedenfalls ist es sinnvoller, sie gezielt und systematisch in den Systementwicklungsprozess einzubringen und sich in diesem Bereich zu bewegen.

Bei der Suche nach Lösungen für dieses Problem sind Anforderungsmuster ein hilfreiches Instrument. Solche Muster sind eine bewährte Unterstützung bei der Entwicklung von Systemen. ${ }^{4}$ Sie helfen Softwareentwicklern, indem sie häufig auftretende Problemstellungen beschreiben und den Kern der möglichen Lösungen darlegen. In der Literatur werden Muster häufig als „Schablonen“ etablierter Lösungen für häufig wiederkehrende Probleme der Systementwicklung bezeichnet. Ein Muster legt damit die Grundstruktur einer Lösung für ein spezielles Problem fest, beschreibt aber noch keine vollständige Lösung des Problems.

\section{Ansätze für die Lösung von Zielkonflikten}

Wenn nicht eine Anforderung hinter einer anderen vollständig zurückstehen soll, muss versucht werden, sie in der Gestaltung des informationstechnischen Systems zu harmonisieren. Durch geeignete Gestaltung kann ein SPA z. B. Anforderungen der Funktionalität, der Dienstleistungsqualität und des Datenschutzes gemeinsam erfüllen.

Um konfliktäre Anforderungen unter Einbezug des Datenschutzes zu lösen, gibt es diverse Ansätze, die sowohl in der Praxis als auch in der Forschung untersucht und eingesetzt werden. Dazu gehören die Ansätze des Privacy by Design, des Privacy by Default und der Gewährleistung von Transparenz der Datenverarbeitung und Entscheidungsfreiheit.

Der Ansatz des Privacy by Design zielt auf eine datenschutzgerechte Systemgestaltung. ${ }^{5}$ Dieser Ansatz zur Umsetzung datenschutzrechtlicher Anforderungen wird jetzt auch seit 2018 von Art. 25 Abs. 1 DSGVO anerkannt. Diese Vorschrift fordert vom Verantwortlichen, „geeignete technische und organisatorische Maßnahmen - wie z. B. Pseudonymisierung - (zu treffen), die dafür ausgelegt sind, die Datenschutzgrundsätze wie etwa Datenminimierung wirksam umzusetzen und ... die Rechte der betroffenen Personen zu schützen ". Indem sie bei dieser datenschutzgerechten Systemgestaltung den Stand der Technik, die Implementierungskosten und die Art, den Umfang, die Umstände und die Zwecke der Verarbeitung sowie die unterschiedliche Eintrittswahrscheinlichkeit und Schwere der mit der Verarbeitung verbundenen Risiken für die Rechte und Freiheiten natürlicher Personen berücksichtigt wissen will, ist sie in der Umsetzung offen für Kompromisse mit anderen Anforderungen an das System. Die Anforderung der datenschutzgerechten Systemgestaltung richtet

4 Alexander, A Pattern Language: Towns, Buildings, Construction, 1977.

5 Roßnagel, DuD 1999, 253 ff.; Spiekermann, Communications of the ACM 2012, 38.

6 Siehe Hansen, in: Simitis/Hornung/Spiecker, Datenschutzrecht, 2019, Art. 25 Rn. 28 ff. die DSGVO allerdings nur an den Verantwortlichen, nicht an den Systemhersteller, der eigentlich ihr Adressat sein müsste. ${ }^{7}$ Ein geeignetes Gestaltungsinstrument ist eine datensparsame Systemarchitektur, in der der Datenfluss so organisiert ist, dass der Verantwortliche nur diejenigen Daten speichert, die für die Zweckerfüllung unbedingt notwendig sind. Der Speicherort der Daten wird hier je nach Sensitivität der Daten gewählt. Soweit möglich, werden sensitive Daten auf dem Endgerät des Nutzers verarbeitet und gespeichert und nur die Daten bei Verantwortlichen oder in einer Cloud, für die dies nicht möglich ist oder die als weniger sensitiv gelten.

Eng verwandt mit dem Ansatz der datenschutzgerechten Systemgestaltung ist der Ansatz des „Privacy by Default“. Dieser ist in Art. 25 Abs. 2 DSGVO als rechtliche Anforderung anerkannt. ${ }^{8}$ Danach hat der Verantwortliche ,geeignete technische und organisatorische Maßnahmen (zu treffen), die sicherstellen, dass durch Voreinstellung grundsätzlich nur personenbezogene Daten, deren Verarbeitung für den jeweiligen bestimmten Verarbeitungszweck erforderlich ist, verarbeitet werden. Diese Verpflichtung gilt für die Menge der erhobenen personenbezogenen Daten, den Umfang ihrer Verarbeitung, ihre Speicherfrist und ihre Zugänglichkeit.“ Diese Anforderung steht nicht unter dem Vorbehalt konkurrierender Anforderungen. Da aber die Erforderlichkeit sich am Verarbeitungszweck orientiert, werden alle Anforderungen, die mit dem Zweck zusammenhängen, berücksichtigt. ${ }^{9}$ Dadurch entstehen auch hier Möglichkeiten der Konfliktlösung durch Gestaltungsentscheidungen. Grundsätzlich muss der Verantwortliche die Voreinstellungen des Systems möglichst datensparsam gestalten und es dem Nutzer überlassen, diese Einstellungen individuell anzupassen, wenn er zusätzliche Funktionen nutzen will. ${ }^{10}$ Dadurch startet der Nutzer mit einer möglichst hohen Datenschutzeinstellung und übersieht keine datenkritischen Voreinstellungen.

Der dritte hier zu besprechende Gestaltungsansatz verknüpft ausreichende Transparenz mit Entscheidungsfreiheit. Sind die kritischen Funktionen des SPA getrennt konfigurier- und nutzbar, und nicht in Bundles zusammengefasst, kann der Nutzer selbst entscheiden, welche er nutzen und für welche er die notwendigen Datenverarbeitungen zulassen will. Voraussetzung für diese freie Entscheidung ist jedoch, dass er rechtzeitig, situationsadäquat und umfassend über die datenschutzrechtlichen Risiken und Konsequenzen informiert wird. ${ }^{11}$ Dieser Ansatz unterstützt durch Aufklärung und Wahlmöglichkeiten die informationelle Selbstbestimmung und trägt darüberhinausgehend auch zum Vertrauen in das Informationssystem bei.

Die vorgestellten Ansätze zur Lösung des Konflikts konkurrierender Anforderungen setzen jedoch voraus, dass die Anforderungen präzise und korrekt erhoben und ihre konfliktären Aspekte ausreichend genau herausgearbeitet sind. Ein wichtiger Faktor, der für den Erfolg der Konfliktlösung entscheidend ist, liegt daher bereits in der Anforderungserhebung. Die Qualität und Formulierung der erhobenen Anforderungen haben einen

\footnotetext{
7 Siehe kritisch Roßnagel/Geminn, Datenschutzgrundverordnung verbessern, 2020, $91 \mathrm{ff}$.

8 Hansen (Fn. 6), Art. 25 Rn. 39.

9 Siehe hierzu Roßnagel, in: Simitis/Hornung/Spiecker, Datenschutzrecht, 2019, Art. 5 Rn. 122 ff.

10 Schomberg/Barev/Janson, DuD 2019, 774-780.

11 Siehe z. B. Husemann, Notwendige Transparenz für betroffenen Personen, in: Roßnagel/Hornung, Grundrechtsschutz im Smart Car, 2019, 367 ff.
} 
Einfluss auf die daraus resultierende Umsetzung im System. Für die relevante Anforderungserhebung können Anforderungsmuster hilfreich sein.

\section{Anforderungsmuster}

Die Anforderungserhebung ist ein wichtiger, aber auch kritischer Schritt für den darauffolgenden Systementwicklungsprozess. Nur wenn die Anforderungen explizit und widerspruchsfrei formuliert werden, können sie für den weiteren Entwicklungsprozess als Grundlage dienen. Dem Prozess der Anforderungserhebung liegen insbesondere Wünsche und Anforderungen des Auftraggebers zugrunde. Der Anforderungsanalyst muss aber auch die Anforderungen berücksichtigen, die der Auftraggeber zwar nicht ausdrücklich formuliert hat, die er aber berücksichtigen muss, wenn er in soziotechnischen Systementwicklungsprojekten am Ende ein akzeptiertes und akzeptables IT-System nutzen will. Rechtliche Anforderungen oder anerkannte Anforderungen der Dienstleistungsqualität gehören zu solchen unabdingbaren Systemanforderungen.

Zur Qualität der Anforderungen gehört es Unklarheiten und Zweideutigkeiten zu vermeiden. Die Anforderungsanalyse sollte für die Systementwicklung klare Bedingungen liefern. Gerade in soziotechnischen Systementwicklungsprojekten kommt es leicht zu unterschiedlichen Verständnissen bei der Anforderungserhebung sowie zu unklaren oder gar unerkannten Konflikten zwischen verschiedenen Anforderungen.

Um Anforderungsanalysten und Systementwickler bei dem Schritt der Anforderungserhebung zu unterstützen, hat sich der Einsatz von Anforderungsmustern bewährt. ${ }^{12}$

\subsection{Definition}

Anforderungsmuster verfolgen das Ziel, eine Sammlung von Wissen und Erfahrungen zu schaffen, die Softwareentwicklern helfen soll wiederkehrende Probleme zu lösen. ${ }^{13}$ Sie beschreiben bewährte Lösungen für immer wieder auftretende Probleme. Anforderungsmuster stellen etablierte Werkzeuge dar, um komplexes Wissen für Anforderungsanalysten und Systementwickler zugänglich und somit auch anwendbar zu machen. Anforderungsmuster decken nicht alle denkbaren Problembereiche ab, da sie Lösungen nur für immer wiederauftretende Probleme bereitstellen. Aufgrund ihrer Abstraktion lösen sie in der Regel Probleme unterschiedlicher Klassen von Systemen durch gemeinsame Anforderungen. ${ }^{14}$

Erfahrungsgemäß entstehen bei der Anforderungserhebung wiederkehrende Probleme mit etablierten Lösungsansätzen. Diese greifen Anforderungsmuster und liefern für sie eine im Entwicklungsprozess brauchbare Beschreibung der Anforderungen, mit denen wiederkehrende Probleme in Form von konkreten konstruktiven Anforderungen an das System erfasst werden können. Solche wiederkehrenden Probleme können z. B. aus dem Recht kommen, weil durch eine bestimmte technische Funktion immer wieder ein Verstoß gegen rechtliche Vorgaben erfolgen

12 Hoffmann/Schulz/Zirfas et al, Legal Compatibility as a Characteristic of Sociotechnical Systems, Business Information Systems, 2015.

13 Alexander (Fn 4).

$14 \mathrm{Gamma/Helm} / \mathrm{Johnson}$ et al., Design Patterns. Elements of Reusable Object-Oriented Software, 1994 kann oder eine besondere rechtliche Anforderung erfüllt werden muss. Gleichzeitig können aber auch stabile Lösungsaspekte in Betracht gezogen werden, die sich mit der qualitätszentrierten Gestaltung von Informationssystemen befassen. Anforderungsmuster sind besonders wertvoll, wenn sie sich Problemen widmen, für deren Erkennen und Lösen spezielles Domänenwissen notwendig ist. Insbesondere bei Anforderungen an die Rechtsverträglichkeit stehen Anforderungsanalysten vor einigen Herausforderungen, welche beispielsweise auf mangelndes Fachwissen zurückzuführen sind. In diesen Fällen sollten Anforderungsmuster dieses Wissen für alle Anspruchsgruppen übergreifend zugänglich machen.

\subsection{Entwicklung der Anforderungsmuster}

Die Entwicklung der Anforderungsmuster für die Anforderungserhebung rechtlicher und qualitativer Anforderungen basiert auf verschiedenen Methoden. Da in der Praxis separate Anforderungserhebungen je Disziplin üblich sind, werden für beide Disziplinen, Recht und Dienstleistungsqualität, eigene Anforderungsmuster erstellt. Damit werden gleichzeitig die einleitend im ersten Abschnitt erwähnten Zielkonflikte transparent gemacht und übergeordnete Ziele in Bezug auf das Erreichen von Dienstleistungsqualität und Rechtsverträglichkeit extrahiert, welche für die Kombination aller Anforderungsmuster genutzt werden können.

Die Erstellung von Anforderungsmustern erfolgt in der Praxis durch damit beauftragte Anforderungsanalysten oder durch die Gemeinschaft der Systementwickler, die sich zu Anforderungen und Gestaltungsvorschlägen auf einschlägigen Plattformen austauschen. Meist fehlt es an Ressourcen für die systematische Erstellung von Anforderungen. Sie entstehen daher vielfach ohne Domänenwissen und methodische Absicherungen aus der Dokumentation von wiederholten und als bewährt geltenden Praxisschritten. Einmal erstellt, haben sie jedoch den Vorteil, dass sie u. U. vieltausendmal verwendet werden können.

Im DFG-Forschungsprojekt „Anforderungs- und Entwurfsmuster zur rechtsverträglichen und qualitätszentrierten Entwicklung kontextsensitiver Applikationen" (AnEkA) konnten für die Gestaltungskriterien der Dienstleistungsqualität und der Rechtsverträglichkeit mit Domänenwissen methodisch hergeleitete Anforderungsmuster erstellt werden, um sie als Instrumente für die Konfliktlösung zwischen konkurrierenden Anforderungen an SPAs zu erproben.

Für die Erstellung der Anforderungsmuster werden sowohl Erkenntnisse aus der einschlägigen Literatur als auch Erkenntnisse und Einblicke aus der Praxis verwendet. Die Erstellung der Anforderungsmuster unterscheidet sich inhaltlich dennoch grundlegend zwischen Disziplinen, hier für Recht und Dienstleistungsqualität.

Für die Erstellung der Anforderungsmuster der Dienstleistungsqualität wurden zunächst Fokusgruppen mit Anforderungsanalysten und Systementwicklern durchgeführt. Im Rahmen der Fokusgruppen wurden bekannte Problematiken und bewährte Lösungsansätze für diese Probleme erhoben und konsolidiert zusammengetragen. Ergänzend zu den Einblicken der Praxis wurde eine Literaturrecherche durchgeführt. Neben bewährten Lösungsansätzen unterstützt die Literaturrecherche die Identifizierung von Kategorien für die Anforderungsmuster. Damit die Anforderungsmuster auf möglichst viele Systeme angewendet werden können, muss eine Allgemeingültigkeit und Genera- 
Abbildung 1 | Beispielhaftes Anforderungsmuster der Rechtsverträglichkeit

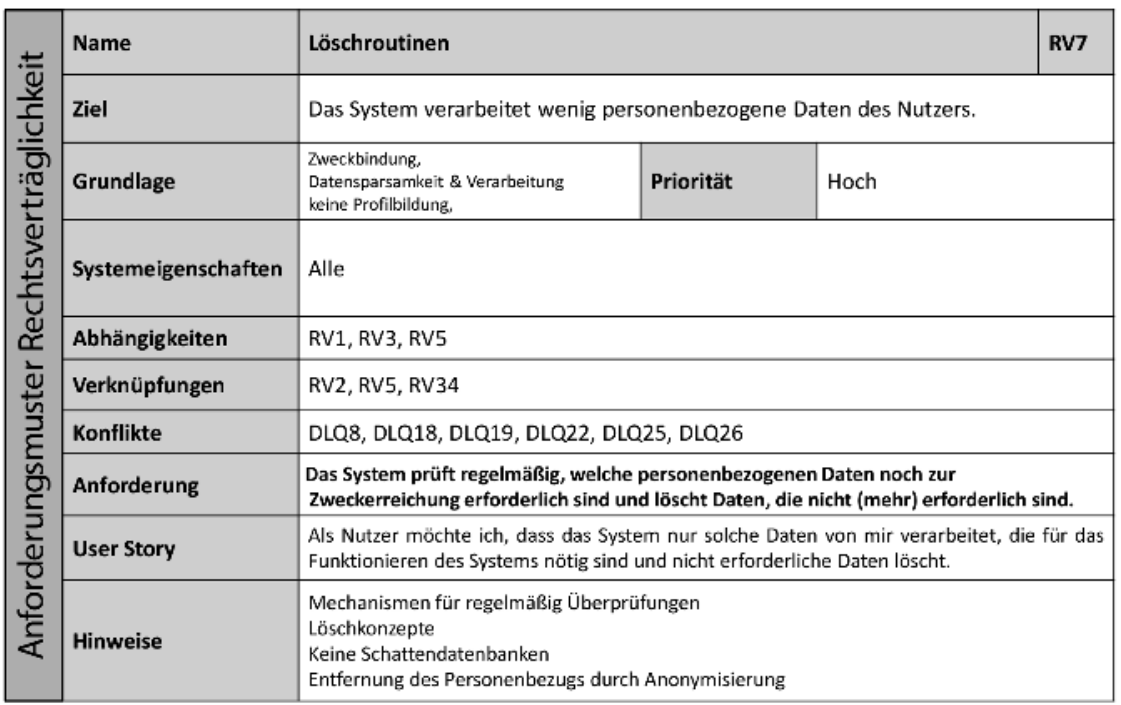

in Form einer optimalen, mit anderen Anforderungen abgestimmten Umsetzung. ${ }^{19}$

\subsection{Aufbau der Anforderungsmuster}

Der Aufbau der Anforderungsmuster soll möglichst interdisziplinär gestaltet werden, sodass diese von beiden Disziplinen gleichermaßen genutzt werden können und die Anforderungserhebung unterstützt wird. Wie bei Anforderungsmustern in der Systementwicklung üblich, empfiehlt es sich, für interdisziplinäre Anforderungsmuster eine tabellarische Darstellung zu nutzen. Diese ermöglicht, viele Informationen auf kleinem Raum übersichtlich darzustellen, ohne dass die Übersicht als auch die Nutzbarkeit der Anforderungsmuster leidet. ${ }^{20}$ Dafür wurden verschiedene Kategorien erstellt, die sowohl in den Anforderungsmustern der lisierbarkeit der Muster gegeben sein. Daher wurden die Inhalte der Anforderungsmuster in einem abschließenden Schritt abstrahiert und so für die Anforderungserhebung verschiedener Klassen von Systemen nutzbar gemacht.

Die rechtlichen Anforderungsmuster hingegen wurden aus abstrakten grundrechtlichen Vorgaben abgeleitet. ${ }^{15}$ Vorteil des Rückgriffs auf diese Vorgaben ist es, dass sie im Gegensatz zu einfachgesetzlichen Vorgaben, die häufiger Änderungen unterliegen, sehr stabil sind und die entsprechenden Muster daher unabhängig von Gesetzesänderungen langfristig einsetzbar sind. Um zu Anforderungen für die rechtsverträgliche Gestaltung von SPA zu gelangen, wurden ausgehend von spezifischen Chancen und Risiken, die SPA mit sich bringen, zunächst die einschlägigen grundrechtlichen Vorgaben identifiziert. ${ }^{16} \mathrm{Um}$ aus diesen abstrakten Vorgaben konkrete rechtliche Anforderungen für SPA zu generieren, wurden aus den grundrechtlichen Vorgaben in mehreren Schritten konkrete Anforderungen abgeleitet. ${ }^{17}$ So lässt sich etwa aus Art. 7 GRCh, der unter anderem das Recht auf Achtung des Privatlebens normiert, ${ }^{18}$ ableiten, dass SPA derart gestaltet werden müssen, dass sie nicht in die Privatsphäre der Nutzer eingreifen. Aus dieser Anforderung lassen sich wiederum mehrere rechtliche Kriterien ableiten. Das sind in diesem Fall z. B. „Kernbereichsschutz", „Zweckbindung“ und „Keine Profilbildung“. Diese Kriterien werden in den Anforderungsmustern als Grundlagen bezeichnet. Sie sind wesentlich konkreter als die zuvor beschriebenen rechtlichen Vorgaben selbst. In Kombination mit den in den Anforderungsmustern verfügbaren Hinweisen zur technischen Umsetzung geben sie Entwickler konkrete Vorschläge zur rechtsverträglichen Gestaltung eines SPA. Ziel bei dieser Vorgehensweise ist nicht nur die Rechtmäßigkeit, also die minimale Einhaltung aller gesetzlichen Vorgaben zur Vermeidung von Sanktionen, sondern die Rechtsverträglichkeit der Sprachassistenten

15 Roßnagel, Methoden, KORA, in: Roßnagel/Hornung/Geminn/Johannes, Rechtsverträgliche Technikgestaltung und technikadäquate Rechtsentwicklung, 2018, $77 \mathrm{ff}$

16 Knote/Thies/Söllner et al., Informatik Spektrum, 2020, 118 (121).

17 Roßnagel (Fn 15).

18 Kingreen, in: Callies/Ruffert EUV AEUV 2016, Art. 7 GRCh, Rn. 3
Rechtsverträglichkeit als auch in denen der Dienstleistungsqualität genutzt werden.

So sind die Kategorien Name, Ziel, Grundlage, Systemeigenschaften, Abhängigkeiten, Verknüpfungen, Konflikte, Anforderung, User Story, Hinweise und Priorität entstanden. Die elf Kategorien werden durch einen eindeutigen Namen des Anforderungsmusters ergänzt. Der eindeutige Name repräsentiert den Inhalt des Musters und impliziert somit auch das Ziel der Anwendung des Musters.

Dadurch, dass es Anforderungsmuster sowohl für die Rechtsverträglichkeit als auch für die Dienstleistungsqualität gibt, können Anforderungen beider Disziplinen getrennt voneinander, aber dennoch in einem gemeinsamen Schritt erhoben werden. Dadurch entstanden zwei, zunächst isoliert voneinander stehende, Anforderungsmuster, die gemeinsam in einem Anforderungsmusterkatalog gesammelt sind. Dabei folgen alle Anforderungsmuster demselben Aufbau.

Jedes Anforderungsmuster wird in einer einseitigen Darstellung präsentiert, wodurch alle notwendigen Informationen auf einen Blick verfügbar sind und nicht viele verschiedene Seiten gelesen werden müssen, um die gesuchte Information zu finden. Abbildung 1 zeigt ein beispielhaftes Anforderungsmuster der Rechtsverträglichkeit. Das Anforderungsmuster „Löschroutinen " thematisiert die Datenverarbeitung im System und schlägt die Anforderung „Das System prüft regelmäßig, welche personenbezogenen Daten noch zur Zweckerreichung erforderlich sind, und löscht Daten, die nicht mehr erforderlich sind" vor. Ergänzend zu dem Namen und der Anforderung enthält das Anforderungsmuster weitere Felder, die die Anwendung und das Verständnis des Musters unterstützen sollen. Im Ziel wird der ideale Zustand durch die Anwendung des Anforderungsmusters abgebildet. Das Ziel ist möglichst kurz und verständlich beschrieben und besteht aus maximal zwei Sätzen. Da jede Anforderung für die praktische Umsetzung priorisiert werden muss, existiert das Feld Priorität, in dem ein drei stufiges Prioritätensystem ab-

19 Siehe zum Konzept der Rechtsverträglichkeit Roßnagel, Rechtswissenschaftliche Technikfolgenforschung, 1993, $189 \mathrm{ff}$.

20 Für Veröffentlichung akzeptiert: Dickhaut/Janson/Leimeister, Codifying Interdisciplinary Design Knowledge through Patterns, DESRIST, 2020. 
Abbildung 2 | Beispielhaftes Anforderungsmuster der Dienstleistungsqualität

\begin{tabular}{|c|c|c|c|c|c|}
\hline$:$ & Name & \multicolumn{3}{|c|}{ Lernen durch Relevanzbewertungen } & DLQ18 \\
\hline$\frac{\bar{\pi}}{\frac{\pi}{\partial}}$ & Ziel & \multicolumn{4}{|c|}{$\begin{array}{l}\text { Der Nutzer erhält, abhängig von seinen Relevanzbewertungen, auf Dauer für ihn } \\
\text { relevantere Informationen. }\end{array}$} \\
\hline ถู & Grundlage & $\begin{array}{l}\text { Lernfähigkeit } \\
\text { Personalisierung }\end{array}$ & Priorität & Mittel & \\
\hline$\frac{n}{4}$ & Systemeigenschaften & \multicolumn{4}{|c|}{ Adaptivität: adaptives Verhalten } \\
\hline$\frac{c}{2}$ & Abhängigkeiten & \multicolumn{4}{|l|}{ DLQ6 } \\
\hline 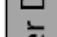 & Verknüpfungen & \multicolumn{4}{|l|}{ RV14, RV18 } \\
\hline$\stackrel{n}{n}$ & Konflikte & \multicolumn{4}{|l|}{ RV5, RV6, RV7 } \\
\hline $\begin{array}{l}\frac{\varepsilon}{\mathrm{n}} \\
\frac{\xi}{丂}\end{array}$ & Anforderung & \multicolumn{4}{|c|}{$\begin{array}{l}\text { Das System soll auf Basis der Relevanzbewertungen des Nutzers mit } \\
\text { fortschreitender Nutzungsdauer relevantere Informationen für den Nutzer } \\
\text { ausgeben. }\end{array}$} \\
\hline$\frac{\overline{1}}{0}$ & User Story & \multicolumn{4}{|c|}{$\begin{array}{l}\text { Als Nutzer möchte ich passendere Informationen erhalten, nachdem ich die } \\
\text { Relevanz vergangener Informationen bewertet habe, um auf Dauer ein } \\
\text { individuelleres Erlebnis zu bekommen. }\end{array}$} \\
\hline$<$ & Hinweise & \multicolumn{4}{|c|}{ Häufige Bewertungen können die Interaktion mit dem System unterbrechen. } \\
\hline
\end{tabular}

einen schnellen Überblick farblich markiert ist. Zudem befindet sich in der rechten oberen Ecke eine eindeutige ID des Musters, die durch RV für Rechtsverträglichkeit und DLQ für Dienstleistungsqualität repräsentiert wird.

Die Anforderungsmuster unterstützen die Anforderungserhebung. Durch konfliktäre Anforderungen können in der praktischen Umsetzung der Anforderungen Konflikte entstehen, die eine kreative Lösungsfindung erfordern. Diese führen zu Gestaltungsvorschlägen, die allen konfligierenden Anforderungen gerecht werden. Sie können in Entwurfsmustern beschrieben werden. ${ }^{21}$

\section{Schlussfolgerungen}

Rechtsverträglichkeit und Dienstleistungsqualität sind wichtige Kriterien für gebildet wird. Die Prioritäten können niedrig, mittel oder hoch sein. Da die Anforderungen in der Praxis nicht isoliert voneinander existieren, stellen die Felder Abhängigkeiten, Verknüpfungen und Konflikte möglicher Interaktionen zwischen den einzelnen Anforderungsmustern dar. Diese können sowohl zwischen Anforderungsmustern der Rechtsverträglichkeit als auch disziplinübergreifend zwischen Anforderungsmustern der Dienstleistungsqualität und Rechtsverträglichkeit vorkommen. Um die Hintergründe der Anforderungsmuster abzubilden, wurde das Feld Grundlage eingeführt. Ergänzend zu den teilweise theoretischen Informationen stellt das Feld User Story die Sicht des Endanwenders dar und hilft das Muster in der Praxis einzuordnen. Für die technische Umsetzung des Musters sind die Felder Systemeigenschaften und Hinweise von Bedeutung, die dem Anwender technische Details bieten.

In Abbildung 2 ist ein Anforderungsmuster der Dienstleistungsqualität zu sehen. In der Erstellung der Anforderungsmuster wurde Wert daraufgelegt, dass die Anforderungsmuster beider Disziplinen sich im Kern nicht stark voneinander unterscheiden. Dennoch muss die Disziplin des jeweiligen Musters auf einem Blick erkennbar sein. Diese wurde durch zwei Details erreicht. Im linken Bereich des Musters steht die Disziplin, die für die Erhebung von Anforderungen an SPA. Zwischen beiden Kriterien gibt es zahlreiche potenzielle Zielkonflikte, für deren Erfassung der Einsatz von Anforderungsmustern in der Anforderungserhebung hilfreich ist. Um solche Zielkonflikte aufzulösen und SPA qualitativ hochwertig wie auch rechtsverträglich zu gestalten, ist es erforderlich, zunächst die Anforderungen aus beiden disziplinären Kriterien in systematischer Weise mithilfe von Anforderungsmustern zu erheben. So können Konflikte, Verbindungen und Synergien identifiziert werden. Die Verwendung von Mustern zeigt positive Auswirkungen auf die Entwicklungseffizienz, die Zusammenarbeit zwischen den Beteiligten und die Qualität von Anforderungsspezifikationen und zu entwickelnden Systemen. ${ }^{22}$ In einem nächsten Schritt können dann mit Entwurfsmustern die identifizierten Zielkonflikte aufgelöst werden.

Anforderungsmuster der hier beschriebenen Art sind weitgehend verallgemeinerbar, sie können für viele Systeme adaptiert werden und können über das Beispiel der SPA hinaus helfen, akzeptable und akzeptierte Technik zu entwickeln.

21 Siehe zu diesen Thies/Dickhaut/Janson et al., in diesem Heft.

22 Hoffmann, Anforderungsmuster zur Spezifikation soziotechnischer Systeme. Standardisierte Anforderungen der Vertrauenswürdigkeit und Rechtsverträglichkeit, 2014. 